\title{
METALS IN THE ICM: WITNESSES OF CLUSTER FORMATION AND EVOLUTION
}

\author{
Lorenzo Lovisari ${ }^{a, *}$, TAtiana F. LAganá ${ }^{b}$, KATHARina Borm $^{a}$, \\ Gerrit Schellenberger ${ }^{a}$, Thomas H. Reiprich ${ }^{a}$ \\ a Argelander-Institut für Astronomie, Auf dem Hügel 71, D-53121 Bonn, Germany \\ ${ }^{b}$ Universidade de São Paulo, Instituto de Astronomia, Geofísica e Ciências Atmosféricas, Departamento de \\ Astronomia, Cidade Universitária, CEP:05508-090, São Paulo, SP, Brazil \\ * corresponding author: lorenzo@astro.uni-bonn.de
}

\begin{abstract}
The baryonic composition of galaxy clusters and groups is dominated by a hot, X-ray emitting Intra-Cluster Medium (ICM). The mean metallicity of the ICM has been found to be roughly $0.3 \div 0.5$ times the solar value, so a large fraction of this gas cannot be of purely primordial origin. Indeed, the distribution and the amount of metals in the ICM is a direct consequence of the past history of star formation in the cluster galaxies and of the processes responsible for the injection of enriched material into the ICM. We here briefly summarize current views on the chemical enrichment, focusing on the observational evidence in terms of metallicity measurements in clusters, spatial metallicity distribution and evolution, and expectations from future missions.
\end{abstract}

KEYwORDS: galaxies: cluster: general, galaxies: abundances, X-ray: galaxies: cluster: intergalactic medium.

\section{INTRODUCTION}

In recent decades a strong effort has been made to shed light on the metal enrichment processes in galaxy clusters, but although interesting results have been obtained we are still far from a full understanding of the enrichment history of these clusters.

Thanks to their deep potential wells, galaxy clusters and groups retain most of the gas released by the member galaxies, making them excellent laboratories for the study of the metal enrichment. The accumulation of the enriched material in clusters can be used as a probe of: a) the source of metals; b) the mechanisms that injected the elements into ICM; c) the different importance of various classes of $\mathrm{SNe}$; d) the star formation history of the Universe. In this context, the study of galaxy groups is of extreme importance, because low mass systems are the most effective environment for the conversion of baryons into stars (e.g. [10]).

All elements apart from Hydrogen and Helium have been synthesized by the stars, which reside mainly in galaxies. In particular, $\mathrm{C}$ and $\mathrm{N}$ are produced by intermediate mass stars, $\mathrm{O}, \mathrm{Ne}, \mathrm{Mg}$ are mainly produced by core-collapse $\mathrm{SNe}$, and $\mathrm{Fe}$ and $\mathrm{Ni}$ by SN Ia. The intermediate $\alpha$-elements (e.g. Si, S, Ca, Ar) are produced by both $\mathrm{SNe}$ in similar proportions, so the relative ratio between these elements can provide information about the Initial Mass Function (IMF) shape. Most of the metals that we observe have been produced within the galaxies, and later they have been transported by various interaction processes from the galaxies to the ICM. There is not a single mechanism that always dominates the enrichment, and the effi- ciencies of the processes vary strongly with galaxy and environmental properties. A list of the proposed processes is the following: ram-pressure stripping [12], galactic winds [6, AGN outflows [7, galaxy-galaxy interactions (e.g. [11]), intra-cluster supernovae (e.g. 9]) and gas sloshing (e.g. [18). This list is certainly not complete, and further processes may also contribute in a small fraction to the metal enrichment of the ICM.

Simulations show that all these processes yield abundance distributions with many inhomogeneities that are not dispersed immediately but are gradually spread out (see [20] for a review). Different processes yield different metal distributions and different time dependences of the enrichment. For example, ram-pressure stripping is more efficient at low redshifts and in the center of the clusters, where the densities of the ICM and the galaxy velocity are higher. However, galactic winds are suppressed in the regions of high density.

X-ray spectra are the only measure for the metallicity of the ICM, and can hence give information on the origin of the metals in the clusters. With the first generation of satellites (e.g. ASCA, BeppoSAX), it was just possible to determine the azimuthally averaged metallicity profiles. However, thanks to the high quality of the X-ray observations from of the current generation of satellites it is now also possible to measure in detail the $2 \mathrm{D}$ distribution of the metals in the ICM. 


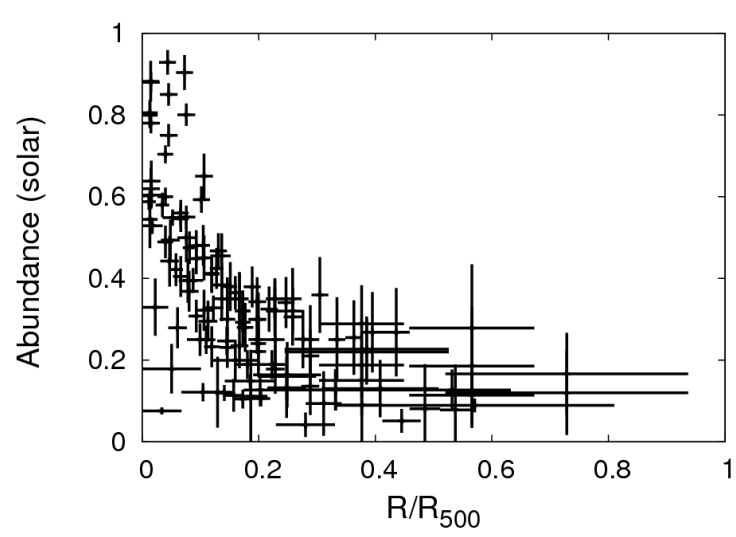

Figure 1. Radial metallicity profiles for 20 galaxy groups with $k T<3 \mathrm{keV}$ (Lovisari et al. in prep.). Abundances are expressed in [1] solar values and radii in units of $R_{500}$.

\section{RADIAL PROFILES}

There is a general consensus that the metal distribution peaks in the center of cool-core clusters, while beyond $\sim 0.2 R_{\text {vir }}$ the metallicity is consistent with being flat, similar to the distribution of non cool-core systems (e.g. 4]). This flattening seems to hold even out to $R_{\text {vir }}[8$. The mean profiles agree well among different instruments (i.e. XMM, Chandra, BeppoSAX, see [13]). Since it is expected that the metals in the ICM originate from stars in galaxies, one would expect the abundance profiles to follow the $\mathrm{cD}$ light profile. However, the light profiles are much steeper than the metal profiles, suggesting that there must be a mixing of the injected metals, a process which may help to diffuse them to larger radii (e.g. [17]).

The abundance profiles of massive clusters have received considerable attention, while the situation in the low mass regime is much less clear, despite their greater importance for the cosmic baryon and metal budgets. In Fig. 1 we show the radial metallicity profiles for a sample of galaxy groups $(k T<3 \mathrm{keV}$, Lovisari et al. in prep.). The profiles have a large scatter, especially at large radii, but they show a universal decrease with radius similar to the decrease observed for massive clusters (see [13]). Although, the overall mean metallicity is $\sim 0.3$, as for hot clusters, and the profiles peak in the center, as for the cool core systems, the abundance at large radii is lower than in massive objects and the intrinsic scatter is most prominent in the core, where non-gravitational processes are expected to be important.

\section{3. $2 \mathrm{D}$ DISTRIBUTION}

Metal profiles are useful for studying central enhanced metallicities due to cool cores or $\mathrm{cD}$ galaxies, on the one hand, and the overall decrease with radius, on the other. The profiles, however, fail to detect the local inhomogeneities of the metals, so a detailed metal map scan would be even more instructive than

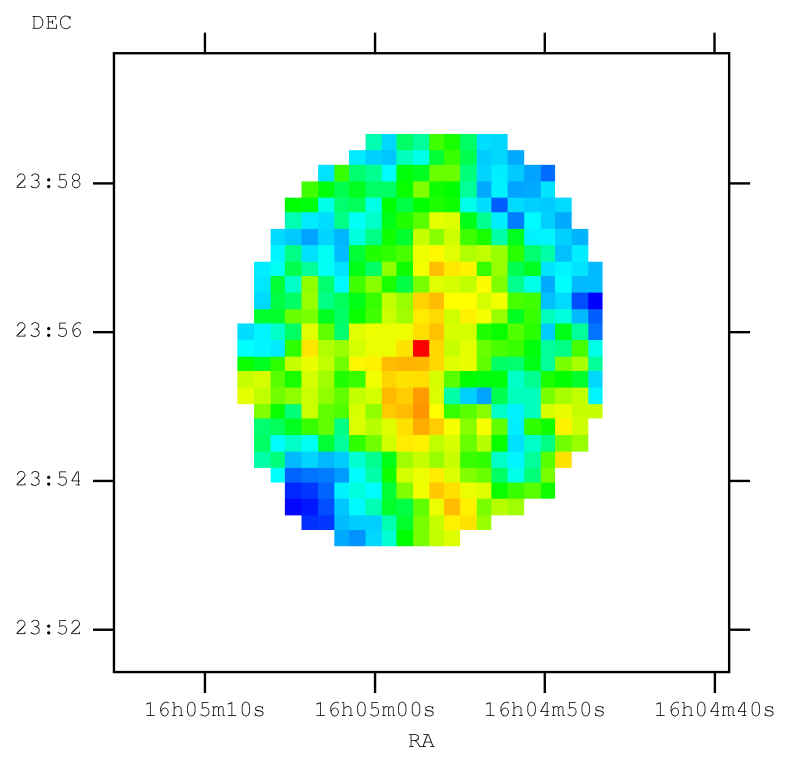

FiguRE 2. Metallicity map of the AWM4 galaxy group, based on spectra extracted from a region centered on, but larger than, the pixels themselves (Laganá et al., in prep.). Although they are not independent, the map can be used for a qualitative study. The colors range from blue $\left(\sim 0.2 Z_{\odot}\right)$ to $\operatorname{red}\left(\sim 0.9 Z_{\odot}\right)$.

the radial profiles. Although it is not easy to derive them, because a lot of photons are required, it was possible to derive detailed metallicity maps for several objects (e.g. [14, 15, 19, 21, 22]). The metallicity distribution appears very inhomogeneous for all the analyzed clusters, even the relaxed clusters, with high metallicity clumps both in the center and in the outskirts (e.g. [15]). Several maxima that are not associated with the cluster center are usually visible in the metallicity distribution. Simulations suggest that these maxima are typically in places where galaxies just have lost a lot of gas. The range of metallicities measured in a cluster from minimum to maximum comprises a factor of at least two or three. The spatial distribution of metals in groups shows a similar non-spherical symmetric distribution as in the clusters (see Fig. 2, Laganá et al. in prep.) suggesting that the same mechanisms are acting at all the mass scales, although their efficiency can deviate for different masses.

\section{Evolution}

In addition to the metal distribution, also the evolution of the metallicity is also interesting. The comparison of the metallicity values between local and high redshift clusters can help to distinguish between the enrichment processes, as different processes have individual time dependences. Furthermore, the observed evolution of metal abundances can trace the star formation rate and the element ratios, e.g. the ratio of $\mathrm{Fe}$ and $\alpha$-elements can be used to obtain information on the different types of supernovae that have 


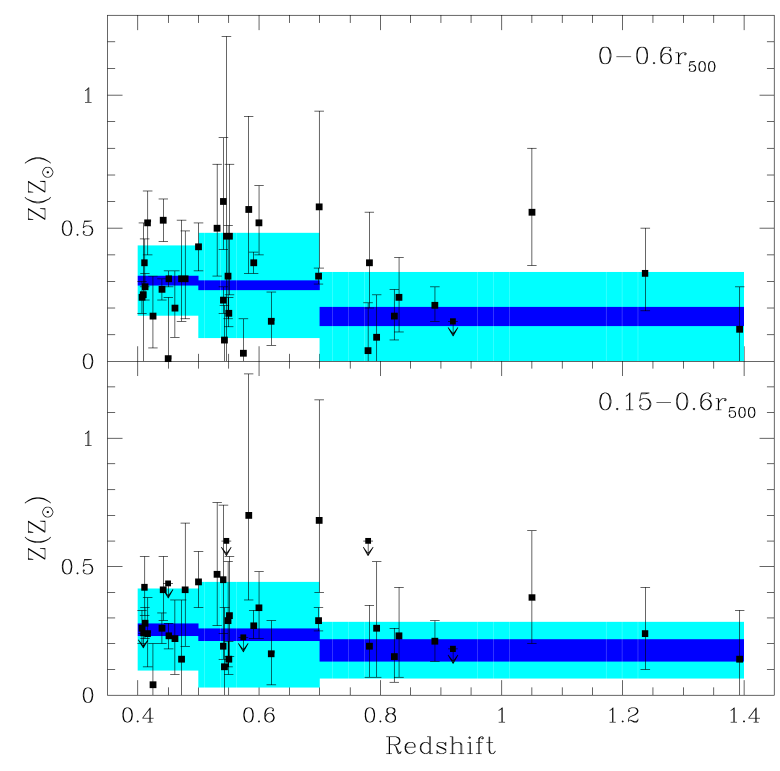

FiguRE 3. Measured abundance as a function of redshift for a sample of 39 clusters, including the emission from the whole cluster at $R<R_{500}$ (top) and excising the core (bottom). The error bars in $Z$ are at $1 \sigma$, while the shaded areas show the weighted mean of the abundance, with its error (blue) and rms dispersion (cyan) in three redshift bins. Although the data are consistent with the no evolution scenario, there is some hint of evolution. Figure adopted from Baldi et al. 2].

contributed to the metal enrichment. It is challenging to measure metal abundances in distant clusters, is challenging, and it requires very high quality data. Only a handful of works (e.g. [2, 3]) have tackled the evolution of metal abundance in the ICM, and the situation remains very clear. Balestra et al. [3] found that the metal abundance changes by a factor of 2 between redshift zero and $\sim 1.3$. A similar evolution of metal abundance with redshift was confirmed by Maughan et al. [16, but recently Baldi et al. [2] failed to find any significant abundance evolution (see Fig. 3).

Since the photon flux is reduced at high redshift, only the Iron-K line emission can be revealed with the current instruments, such that global metallicity and Iron abundance have the same meaning. As we will discuss in the next section, it will be possible in the near future to obtain abundance measurements for different elements up to redshift 0.5 with Astro-H.

\section{Future Missions}

Although eROSITA is not designed for metallicity studies, the large amount of data will enable us to study the metal enrichment as a function of the galaxy cluster mass and redshift. While the low exposures of the individual clusters (a few ks) compared to the study with the current satellites does not allow accurate measurements of the metallicity, the advantage

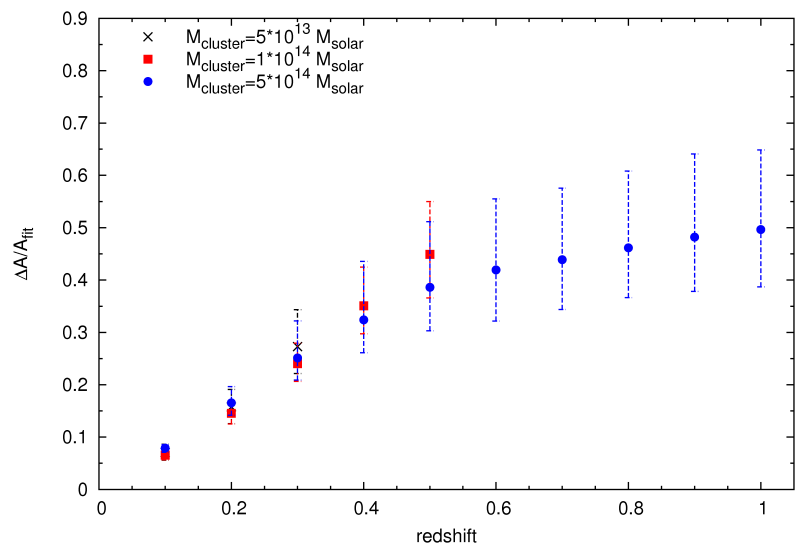

FIgURE 4 . The relative uncertainty on the abundance measurements as a function of redshift and masses for a $20 \mathrm{ks}$ exposure time with eROSITA. The median values for 1000 realizations are shown, and the $68 \%$ errors are taken from the distributions (Borm et al. in prep.).

of eROSITA is the very large number of objects that will be studied. This high statistic together with the dedicated pointed cluster follow-up data will allow us to put constraints on the enrichment processes, on their efficiencies and on the metal evolution with time. In Fig. 4 we show the relative uncertainty on the abundance measurements that we expect to obtain with a $20 \mathrm{ks}$ eROSITA observation (such exposure will be obtained, e.g. around the poles).

On the contrary to eROSITA, one of the Astro-H mission goals is to determines abundances of key diagnostic elements that are currently inaccessible because of their low equivalent widths (i.e., N, Al, Ca, Ar) or blended with the Fe-L shell transitions ( $\mathrm{Ne}$ and $\mathrm{Mg}$ ). In particular, $\mathrm{N}$ is a very good diagnostic of the stellar Initial Mass Function (IMF), while the Ca/Ar ratio is sensitive to the Type Ia explosion mechanism [5]. For the first time, the abundance measurements of $\mathrm{O}, \mathrm{Ne}$, and $\mathrm{Mg}$ will be accurate to $\pm 10 \%$ up to $z \sim 0.2$ and to $\pm 20 \%$ up to $z \sim 0.4$. At the same time, Fe and Si will be determined in bright clusters with a good accuracy even at $z>1$. All these measurements together, will enable us to distinguish between different enrichment mechanisms, to constrain models of the IMF, and to determine key characteristics of Type Ia supernovae, such as their progenitor formation, the explosion mechanisms and their efficiency to enrich the ICM.

\section{Summary}

Measurements of the content, distribution and evolution of the metals in the ICM provide invaluable insights on the interactions between the different components in clusters and about the processes that inject enriched gas into the ICM. While the production of metals is related to the star formation rate, their distribution is associated with many metal enrichment mechanisms that are determined by different physical 
effects. Although the current generation of satellites can measure the distribution of the metals in the ICM with a relatively good accuracy, it is not known yet, which of the processes is most efficient, what the time variations are and how much these factors depend on other parameters like e.g. the mass of the cluster/group. The advent of Astro-H on one side and eROSITA on the other will allow us to measure metal abundance distributions with a very good accuracy and their time evolution, so that we will soon get answers on the importance of the different enrichment processes.

\section{ACKNOWLEDGEMENTS}

LL acknowledges support from the DFG through Heisenberg grant RE 1462/6, from the German Aerospace Agency (DLR) with funds from the Ministry of Economy and Technology (BMWi) through grant 50 OR 1102. THR acknowledges support from the DFG through Heisenberg grant RE $1462 / 5$ and grant RE $1462 / 6$. TFL expresses thanks for financial support from FAPESP (grant 2008/04318-7) and from CAPES (grant BEX 3405-10-9).

\section{REFERENCES}

[1] Asplund, M., Grevesse, N., Sauval, A.: 2009, ARA\&A, 47,481

[2] Baldi, A., Ettori, S., Molendi, S., et al.: 2012, A\&A, 537,142

[3] Balestra, I., Tozzi, P., Ettori, S., et al.: 2007, A\&A, 462,429

[4] De Grandi, S., Ettori, S., Longhetti, M., et al.: 2004, A\&A, 419, 7

[5] de Plaa, J., Werner, N., Bleeker, J.A.M., et al.: 2007, A\&A, 465, 345

[6] De Young, D.S.: 1978, ApJ, 223, 47

[7] De Young, D.S.: 1986, ApJ, 307, 62

[8] Fujita, Y., Tawa, N., Hayashida, K., et al.: 2008, PASJ, 605, 343

[9] Gerhard, O., Arnaboldi, M., Freeman, K.C., et al.: 2002, ApJ, 580, 121

[10] Giodini, S., Pierini, D., Finoguenov, A.: 2009, ApJ, 703,982

[11] Gnedin, N.Y.: 1998, MNRAS, 294, 407
[12] Gunn, J.E., Gott, J.R.: 1972, ApJ, 176, 1

[13] Leccardi, A., Molendi, S.: 2008, A\&A, 487, 461

[14] Lovisari, L., Kapferer, W., Schindler, S., et al.: 2009, A\&A, 508, 191

[15] Lovisari, L., Schindler, S., Kapferer, W.: 2011, 528, 60

[16] Maughan, B.J., Jones, C., Forman, W., et al.: 2008, ApJS, 174, 117

[17] Rebusco, P., Churazov, E., Bhöringer, H., et al.: 2005, MNRAS, 359, 1041

[18] Roediger, E., Lovisari, L., Dupke, R., et al.: 2012, MNRAS, 420, 3632

[19] Sanders, J.S., Fabian, A.C.: 2006, MNRAS, 371, 1483

[20] Schindler, S., Diaferio, A.: 2008, SSRv, 134, 363

[21] Schmidt, R.W., Fabian, A.C., Sanders, J.S.: 2002, MNRAS, 337, 71

[22] Simionescu, A., Werner, N., Bhöringer, H., et al.: 2009, A\&A, 493, 409

\section{Discussion}

Maurice H.P.M. van Putten - In your last-but-one slide, you mentioned your goal of identifying the type Ia $\mathrm{SNe}$ mechanism with astro-H. Does this include identifying their progenitors?

Lorenzo Lovisari - One of the main goals is to provide stringent constraints on the contribution of SN explosions to the metal enrichment of the ICM and the evolution of the SN Ia rate, which is one of the most promising methods for unveiling SN Ia progenitors.

James Beall - Is there an association between the clumpiness you show in the clusters and the central AGN jets?

Lorenzo Lovisari - The metallicity maps show a relation between the direction of highly enriched gas and the cavity/jet angle. Furthermore, there is a strong correlation between the power of the AGN and the maximum radius at which a significant enhancement in metallicity has been detected. 